\title{
Transfer collaborative filtering from multiple sources via consensus regularization
}

\author{
Fuzhen Zhuang $^{\mathrm{a}, \mathrm{b}, *}$, Jing Zheng ${ }^{\mathrm{c}}$, Jingwu Chen ${ }^{\mathrm{a}, \mathrm{b}}$, Xiangliang Zhang ${ }^{\mathrm{d}}$, Chuan Shi $^{\mathrm{c}}$, \\ Qing $\mathrm{He}^{\mathrm{a}, \mathrm{b}}$ \\ ${ }^{a}$ Key Lab of Intelligent Information Processing of Chinese Academy of Sciences (CAS), Institute of Computing Technology, CAS, Beijing 100190, China \\ ${ }^{\mathrm{b}}$ University of Chinese Academy of Sciences, Beijing 100049, China \\ c Beijing University of Posts and Telecommunications, Beijing, China \\ d King Abdullah University of Science and Technology, Thuwal, Saudi Arabia
}

\section{A R T I C L E I N F O}

\section{Article history:}

Received 28 December 2017

Received in revised form 24 July 2018

Accepted 27 August 2018

Available online 5 September 2018

\section{Keywords:}

Collaborative filtering

Transfer learning

Multiple sources

Consensus regularization

\begin{abstract}
A B S T R A C T
Collaborative filtering is one of the most successful approaches to build recommendation systems. Recently, transfer learning has been applied to recommendation systems for incorporating information from external sources. However, most existing transfer collaborative filtering algorithms tend to transfer knowledge from one single source domain. Rich information is available in many source domains, which can better complement the data in the target domain than that from a single source. However, it is common to get inconsistent information from different sources. To this end, we proposed a TRAnsfer collaborative filtering framework from multiple sources via ConsEnsus Regularization, called TRACER for short. The TRACER framework handles the information inconsistency with a consensus regularization, which enforces the outputs from multiple sources to converge. In addition, our algorithm is to learn and transfer knowledge at the same time while most of the traditional transfer learning algorithms are to learn knowledge first and then transfer it. Experiments conducted on two real-world data sets validate the effectiveness of the proposed algorithm.
\end{abstract}

(c) 2018 Elsevier Ltd. All rights reserved.

\section{Introduction}

With the rapid development of E-commerce, people usually make recommendations or predictions for some users by utilizing the known preferences of other users or their own historical behaviors. In recent years, recommendation system, which aims to handle the information overload problem, has attracted vast amount of interest and great deal of research has been devoted to improve recommendation performance (Bobadilla, Ortega, \& Hernando, 2013).

Collaborative filtering is one of the most popular approaches to build recommendation systems (He \& McAuley, 2016; Yang, Lei, Liu, \& Li, 2017). Previous works on collaborative filtering can be grouped into three pipelines according to the information sources they used. First, the pioneering works only utilize the sole source of rating data or check-in data for recommendation (Lee \& Seung, 2001; Srebro \& Jaakkola, 2003). For the second pipeline, to

\footnotetext{
* Corresponding author at: Key Lab of Intelligent Information Processing of Chinese Academy of Sciences (CAS), Institute of Computing Technology, CAS, Beijing 100190, China.

E-mail addresses: zhuangfuzhen@ict.ac.cn (F. Zhuang), ollichooo@gmail.com (J. Zheng), chenjingwu@ict.ac.cn (J. Chen),Xiangliang.Zhang@kaust.edu.sa (X. Zhang), shichuan@bupt.edu.cn (C. Shi), heqing@ict.ac.cn (Q. He).
}

further enhance the recommendation performance, the external information (e.g., users' social networks and items' attributes) is considered by many subsequent algorithms (Diao et al., 2014; Shi et al., 2015; Yu, Che, Li, Li, \& Jiang, 2017). However, external information sometimes is not easy to obtain, and it may not be accurate enough to make the enhancement. The third pipeline is transfer collaborative filtering (TCF) (Fernández-Tobías, Cantador, Kaminskas, \& Ricci, 2012; Gao et al., 2013; Li, Yang, \& Xue, 2009), which tries to learn knowledge from source domains and applies it in the target domain for better recommendation. Our work belongs to this pipeline. Most of the previous TCF algorithms pay attention to transferring knowledge from only one source domain to another. One of the representative work is the cross-domain collaborative filtering method via codebook transfer (CBT) (Li et al., 2009), which assumes different domains may share the same cluster-lever useritem rating pattern called codebook to make knowledge transferred across domains.

However, available information in one source domain may not be enough, and learning knowledge from a single domain is easy to be over-fitting. In addition, in many real-world learning scenarios, auxiliary data can be collected from multiple sources. Although these auxiliary data may follow different data distributions, they 
may be semantically related and share some commonality. Therefore, in this paper, we investigate the problem of transfer collaborative filtering from multiple sources.

Since the knowledge learnt from different sources is used to make predictions for the same target domain, their predicted results should be as similar as possible (Zhuang et al., 2010). Along this line, we design a novel consensus regularization to enforce the predicted results to be similar. Specifically, we propose the transfer collaborative filtering method with consensus regularization (TRACER), which is based on the CBT framework, to transfer knowledge from multiple source domains for collaborative filtering. Most existing TCF methods divide the learning process into two parts, namely learning and transferring (Li et al., 2009; Moreno, Shapira, Rokach, \& Shani, 2012). These TCF methods learn knowledge in various sources firstly and then transfer knowledge to improve the recommendation performance in the target domain. However, the two-stage learning methods cannot adjust the knowledge learning process in the source domains as the prediction in the target domain is not satisfactory. Therefore, our method takes the transfer learning process as a whole and trains the model iteratively, rather than divides the training process into two stages. Our framework not only transfers knowledge from multiple source domains but imposes the consensus regularization on their outputs to force the outputs to be similar simultaneously.

To intuitively illustrate the idea of TRACER, we model the recommendation problem with $s$ source domains and one target domain. Fig. 1 shows a toy example of our method. For $s$ source domains, we can learn different knowledge from them and obtain $s$ predicted matrices. In the process of transfer learning, we take constrains on these matrices simultaneously, which performs like a voting majority, the minority is subject to the major. For example, when we predict a rating in the target domain, we may obtain different predicted results from different source domains (e.g., 5, 4,5 from domains 1,2, 3 respectively). Then, the consensus regularization would take effect and force these predicted results to be the value which is the majority (e.g., 5 of domains 1 and 3). Compared with the previous work (Moreno et al., 2012), which extracts knowledge from multiple source domains and assigns the same weights to each source domain when making predictions, our model makes each source domain contribute differently for different predicted ratings.

Furthermore, we illustrate two experiments to validate the effectiveness of our method. The first experiment is to make predictions on the entertainment website Douban, ${ }^{1}$ which contains movie, music and book rating information. For example, when making movie recommendation, TRACER transfers knowledge from book and music domains to boost the recommendation in movie domain. The second experiment is on Dianping website, ${ }^{2}$ another well-known Chinese social media platform providing reviews of users on items. This website contains rating information of restaurants grouped by different cities. For example, the restaurant rating data are from Beijing, Shanghai, Shenzhen, Suzhou and Hangzhou. We regard the rating information from one city as one domain, then our goal is to make restaurant recommendation by transferring knowledge from domains of other cities. Extensive experiments conducted on the real-world applications validate the effectiveness of our framework.

The rest of our paper is organized as follows. Section 2 introduces the related work. Section 3 details the problem formulation and solution derivation of TRACER. The experimental results are shown in Section 4. Finally, a summary is given in Section 5.

\footnotetext{
1 https://www.douban.com/.

2 http://www.dianping.com/.
}

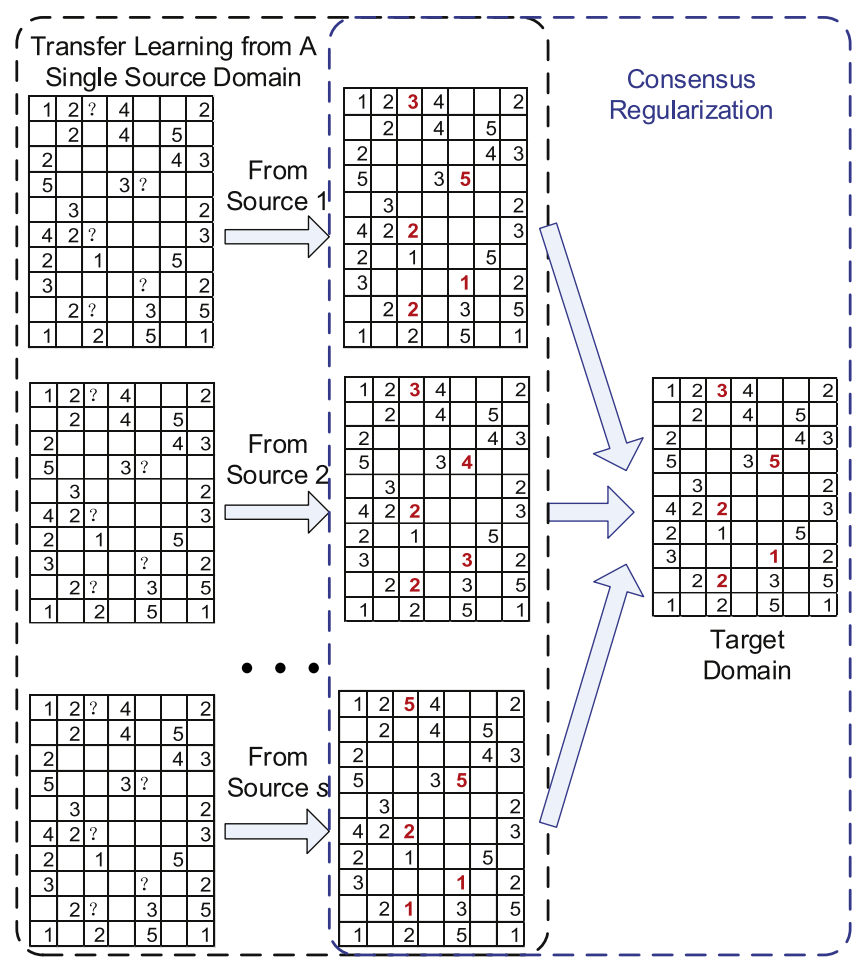

Transfer Collaborative Filtering from Multiple Source Domains to One Target Domain

Fig. 1. The framework of TRACER.

\section{Related work}

According to the utilized information for recommendation systems, we can roughly classify contemporary recommendation methods into three types: rating information (Lee \& Seung, 2001; Mnih \& Salakhutdinov, 2008), external information (Ma, King, \& Lyu, 2009; Shi et al., 2015), and cross-domain information (Chang, Wu, Liu, \& Yang, 2017; Li et al., 2009).

Traditional recommendation methods usually only utilize useritem rating information for recommendation. Collaborative filtering is one of the most popular techniques, which includes two types of approaches: memory-based method and model-based method (Ricci, Rokach, \& Shapira, 2011). Recently, matrix factorization has shown its effectiveness and efficiency in recommendation systems, which factorizes user-item rating matrix into two low rank user-specific and item-specific matrices and then utilizes the factorized matrices to make further predictions (Hernando, Bobadilla, \& Ortega, 2016; Srebro \& Jaakkola, 2003).

In order to address the data sparsity and cold-start problem in traditional recommendations, more and more researches began to utilize different types of external information. With the prevalence of social networks, social information is widely employed in recommendation (Diao et al., 2014; Gu, Sun, \& Gao, 2017; Ma et al., 2009). For example, Ma et al. (2009) fused the user-item matrix with the users' social networks by sharing a common latent lowdimensional user feature matrix. The geographic information is also widely used in Li, Guo, Wang, Li, and Yu (2016), Lian et al. (2014) and Ye, Yin, Lee, and Lee (2011). For example, Lian et al. (2014) augmented users' and POIs' latent factors in the factorization model with activity area vector of users and influence area vector of POIs, respectively. Some approaches also consider time information (Kawamae, 2015; Wang et al., 2015) and the multiple ratings information (Fu, Liu, Ge, Yao, \& Xiong, 2014; He, Chen, 
Kan, \& Chen, 2015; Hong, Doumith, \& Davison, 2013). Recently, more and more researchers have been aware of the importance of heterogeneous information for recommendation. Considering heterogeneous network constructed by different interactions with users, Jamali and Lakshmanan (2013) proposed HETEROMF to integrate a general latent factor and context-dependent latent factors. Shi et al. (2015) proposed the concept of weighted heterogeneous information network and designed a meta-path based recommendation model called SemRec.

In order to integrate more information from different domains for better recommendation, cross-domain recommendation considers combining data from different domains with the original target data (Fernández-Tobías et al., 2012). Transfer learning is widely used for cross-domain recommendation, which aims to leverage the knowledge in the source domain to facilitate the learning tasks in the target domain (Pan \& Yang, 2010). The basic idea of existing methods is to utilize the common latent structure shared across domains as the bridge for knowledge transfer. Shi, Larson, and Hanjalic (2013) proposed a generalized tag induced cross-domain collaborative filtering model through exploiting user-contributed tags as common characteristics. Furthermore, they reviewed two categories of collaborative filtering beyond the user-item matrix: rich side information and interaction information (Shi, Larson, \& Hanjalic, 2014). Recently, considering that the number of overlapped users is usually small, Jiang, Cui, Yuan, Xie, and Yang (2016) proposed a novel semi-supervised transfer learning method to address the problem of cross-platform behavior prediction. Although these cross-domain recommendation methods have achieved success in many applications, these methods usually focus on transferring knowledge from one single source domain to one target domain. However, many applications in real world often involve various domains. Compared to these methods, TRACER utilizes rich information extracted from multiple source domains, which is potential to achieve better performance.

The most related work is the transfer learning for multiple domains method (TALMUD) (Moreno et al., 2012), which generated multiple outputs from different source domains first and then assigned weights to integrate them together. Compared to TALMUD, TRACER learns and transfers knowledge from various source domains simultaneously and forces prediction results for the same missing rating to converge during the learning process. In addition, the two methods have different mechanisms of integrating predicted results. TALMUD assigns the same weight to the predicted ratings learnt from the same source domain, thus there are $s$ different weights if existing $s$ source domains. While TRACER can be regarded as a locally majority voting method, that is, we perform majority voting on each (user, item) pair in the target domain. Therefore, TRACER usually performs better than TALMUD in terms of rating prediction. However, when the predicted ratings for the same missing rating are larger in error, TRACER still performs majority voting and the final integration result may tend to be error. On the other hand, TRACER has a higher time complexity because of its complicated training process.

In all, compared with the previous methods, TRACER utilizes richer information in multiple domains and exploits the consensus regularization to handle the inconsistencies in prediction results generated from different domains. Most existing methods divide the learning process into two parts. They learn knowledge in the source domains first and then transfer it to the target domain, which cannot adjust the learning process in time when the prediction is not satisfactory. The improved learning method of TRACER which trains the model as a whole instead of a two-stage learning process further enhance the recommendation performance.

\section{TRACER framework and its solution}

First, we would like to briefly introduce some notations and denotations which are frequently used in this paper, and they are summarized in Table 1.

\subsection{Problem formalization}

In this section, we formally define the TCF problem with multiple sources and formulate the optimization problem of TRACER. Supposing that there are $s$ auxiliary source domains $\mathcal{D}_{\text {aux }}^{(1)}, \mathcal{D}_{a u x}^{(2)}, \ldots$, $\mathcal{D}_{a u x}^{(s)}$, whose corresponding rating matrices are $R_{a u x}^{(1)}, R_{a u x}^{(2)}, \ldots, R_{a u x}^{(s)}$, and one target domain $\mathcal{D}_{\text {tgt }}$ whose corresponding rating matrix is $R_{t g t}$. The corresponding indicator matrices of these rating matrices are respectively $Q_{a u x}^{(1)}, Q_{a u x}^{(2)}, \cdots, Q_{a u x}^{(s)}$ and $Q_{t g t}$, i.e., $Q_{\operatorname{tgt}(i, j)}=1$ and $Q_{a u x(i, j)}^{(a)}=1(a=1, \ldots, s)$ if user $i$ gave a score to item $j$, else $Q_{\text {tgt }}(i, j)=0$ and $Q_{a u x(i, j)}^{(a)}=0$. Our goal is to leverage the knowledge from these $s$ source domains to improve the recommendation performance in the target domain.

Li et al. (2009) found that users (or items) with similar attributes always behave similar so that they can be clustered into groups in collaborative filtering. Therefore, they presented a concept of "codebook", which is a cluster-level user-item rating pattern of original rating matrix. Furthermore, they proposed the CBT method, which learnt the codebook in source domain and used it as a bridge for knowledge transfer to improve the prediction performance in the target domain. Motivated by the effectiveness of CBT, we adapt CBT as the basic component of our model. It is worth noting that our method takes the transfer learning process as a whole and trains the model iteratively rather than divides the training process into two stages as CBT did. The CBT is illustrated as follows,

$$
\begin{gathered}
\min _{U_{\text {aux }} \geq 0, V_{\text {aux }} \geq 0, U_{\text {tgt }} \geq 0, V_{\text {tgt }} \geq 0, S_{\text {aux }} \geq 0} \mathcal{L}_{1} \\
=\left\|R_{\text {aux }}-U_{\text {aux }} S_{\text {aux }} V_{\text {aux }}^{\top}\right\|^{\top}\left\|^{2}+\right\| R_{\text {tgt }}-U_{\text {tgt }} S_{\text {aux }} V_{\text {tgt }}^{\top} \|^{2},
\end{gathered}
$$

where $U_{\text {aux }} \in \mathbb{R}^{m_{\text {aux }} \times k_{\text {aux }}}$ and $V_{\text {aux }} \in \mathbb{R}^{n_{\text {aux }} \times l_{\text {aux }}}$ are respectively the cluster-level user and item latent factors in source domain, and $U_{\text {tgt }} \in \mathbb{R}^{m_{\text {tgt }} \times k_{\text {aux }}}$ and $V_{\text {tgt }} \in \mathbb{R}^{n_{\text {tgt }} \times l_{\text {aux }}}$ are respectively the cluster-level user and item latent factors in the target domain. $S_{\text {aux }} \in \mathbb{R}^{k_{a u x} \times l_{\text {aux }}}$ is the codebook, which is the bridge for knowledge transfer across two domains. Given $s$ source domains, the optimization problem in Eq. (1) can be rewritten as follows,

$$
\begin{aligned}
& \min _{U_{a u x}^{(a)}, V_{\text {aux }}^{(a)}, U_{\text {tgt }}^{(a)}, V_{\text {tgt }}^{(a)}, S_{a u x}^{(a)} \geq 0, a=1, \ldots, s} \mathcal{L}_{2} \\
& =\frac{1}{S} \sum_{a}\left(\left\|R_{a u x}^{(a)}-U_{\text {aux }}^{(a)} S_{a u x}^{(a)} V_{\text {aux }}^{(a)}\right\|^{\top}\left\|^{2}+\right\| R_{\text {tgt }}-U_{\text {tgt }}^{(a)} S_{\text {aux }}^{(a)} V_{\text {tgt }}^{(a)^{\top}} \|^{2}\right) .
\end{aligned}
$$

Then the remaining challenge is how to combine these $s$ outputs generated by transferring from a single source domain to one target domain. To address this issue, we propose a consensus regularization item to force these $s$ outputs to be similar. Specifically, the second part of TRACER is formulated as,

$$
\begin{aligned}
& \min _{U_{\text {tgt }}^{(a)}, V_{\text {tgt }}^{(a)}, S_{\text {aux }}^{(a)} \geq 0, a=1, \ldots, s} \mathcal{L}_{3} \\
= & \frac{1}{s(s-1)} \sum_{a=b+1}^{s} \sum_{b=1}^{s-1}\left\|U_{\text {tgt }}^{(a)} S_{a u x}^{(a)} V_{\text {tgt }}^{(a)^{\top}}-U_{\text {tgt }}^{(b)} S_{a u x}^{(b)} V_{\text {tgt }}^{(b)^{\top}}\right\|^{2} .
\end{aligned}
$$

This regularization is locally to force the $s$ corresponding predictions on each (user, item) pair in the target domain to make the same result.

Now it is ready to present the optimization problem of TRACER, and we further divide the rating data $R_{\text {tgt }}$ into two parts $R_{\text {tgt }}^{(t r a)}$ and $R_{\text {tgt }}^{(v a l)}\left(R_{\text {tgt }}=R_{\text {tgt }}^{(t r a)} \cup R_{\text {tgt }}^{(v a l)}\right)$, whose corresponding indicator functions are $Q_{\text {tgt }}^{(\text {tra }}$ and $Q_{\text {tgt }}^{(v a l)}\left(Q_{\text {tgt }}=Q_{\text {tgt }}^{\text {(tra })} \cup Q_{\text {tgt }}^{(v a l)}\right) . R_{\text {tgt }}^{(\text {tra })}$ is used for training during transferring from one source domain to one target domain, and $R_{\text {tgt }}^{(v a l)}$ is used as validation set in consensus regularization when 
Table 1

The frequently used notations and denotations.

\begin{tabular}{|c|c|}
\hline Notation & Denotation \\
\hline$R$ & $\begin{array}{l}\text { The rating matrix. Specially, } R_{a u x}^{(a)} \text { denotes the rating matrix of the } a \text { th } \\
\text { source domain, and } R_{\text {tgt }} \text { denotes the rating matrix of the target domain. }\end{array}$ \\
\hline$U, V$ & The latent user/item-cluster indicator matrix. \\
\hline$S$ & The cluster-level user-item rating pattern matrix. \\
\hline Q & The indicator matrix of $R$. \\
\hline$m_{a u x}^{(a)}, n_{a u x}^{(a)}$ & The number of users and items in the $a$ th source domain. \\
\hline$m_{\text {tgt }}, n_{\text {tgt }}$ & The number of users and items in the target domain. \\
\hline$k_{\text {aux }}^{(a)}$ & The number of latent user clusters in the $a$ th source domain. \\
\hline$l_{a u x}^{(a)}$ & The number of latent item clusters in the $a$ th source domain. \\
\hline $\mathbb{R}$ & Nonnegative real number. \\
\hline$U$ & The combination matrix of two rating matrices. \\
\hline T & The transposition of a matrix. \\
\hline$\odot$ & The element-wise product of two matrices. \\
\hline$\oslash$ & The element-wise division of vectors or matrices. \\
\hline
\end{tabular}

performing parameter tuning. Finally, the optimization problem of TRACER is formalized as follows,

$$
\min _{U_{\text {aux }}^{(a)}, V_{\text {aux }}^{(a)}, U_{\text {tgt }}^{(a)}, V_{\text {tgt }}^{(a)},,_{\text {aux }}^{(a)} \geq 0, a=1, \ldots, s} \mathcal{J}=\mathcal{J}_{1}+\alpha \mathcal{J}_{2}+\beta \mathcal{J}_{3},
$$

where

$$
\begin{aligned}
\mathcal{J}_{1}= & \frac{1}{s} \sum_{a=1}^{s}\left\{\| Q_{\text {aux }}^{(a)} \odot\left(R_{\text {aux }}^{(a)}-U_{\text {aux }}^{(a)} S_{\text {aux }}^{(a)} V_{\text {aux }}^{(a)}\right)^{\top}\right) \|^{2} \\
& \left.+\left\|Q_{\text {tgt }}^{(\text {tra })} \odot\left(R_{\text {tgt }}^{(t r a)}-U_{\text {tgt }}^{(a)} S_{\text {aux }}^{(a)} V_{\text {tgt }}^{(a)^{\top}}\right)\right\|^{2}\right\},
\end{aligned}
$$

$\mathcal{J}_{2}=$

$\left.\frac{1}{s(s-1)} \sum_{a=b+1}^{s} \sum_{b=1}^{s-1} \| Q_{\text {tgt }}^{(v a l)} \odot\left(U_{\text {tgt }}^{(a)} S_{a u x}^{(a)} V_{\text {tgt }}^{(a)^{\top}}-U_{\text {tgt }}^{(b)} S_{a u x}^{(b)} V_{\text {tgt }}^{(b)}\right)^{\top}\right) \|^{2}$,

$\mathcal{J}_{3}=\sum_{a=1}^{s}\left(\left\|U_{a u x}^{(a)}\right\|^{2}+\left\|V_{a u x}^{(a)}\right\|^{2}+\left\|U_{\text {tgt }}^{(a)}\right\|^{2}+\left\|V_{\text {tgt }}^{(a)}\right\|^{2}+\left\|S_{a u x}^{(a)}\right\|^{2}\right)$,

$\mathcal{J}_{3}$ is the weight decay item to control the complexity of the proposed framework, $\alpha$ and $\beta$ are the trade-off parameters. $\alpha$ controls the importance of consensus regularization and $\beta$ represents the regularization parameter to avoid over-fitting problem.

\subsection{Solution derivation}

The aim of our framework is to minimize $J$ in Eq. (4) as a function of $U_{a u x}^{(a)}, V_{a u x}^{(a)}, U_{\text {tgt }}^{(a)}, V_{\text {tgt }}^{(a)}$ and $S_{a u x}^{(a)}(a=1, \ldots, s)$. We adopt the gradient descent method to solve this optimization problem, and the detailed derivation can be referred in Appendix.

The updating rules for $U_{a u x}^{(a)}, V_{a u x}^{(a)}, U_{\text {tgt }}^{(a)}, V_{\text {tgt }}^{(a)}$ and $S_{a u x}^{(a)}$ are as follows,

$$
\begin{aligned}
U_{a u x}^{(a)} & \leftarrow U_{a u x}^{(a)} \odot\left\{\left(R_{a u x}^{(a)} V_{a u x}^{(a)} S_{a u x}^{(a)}{ }^{\top}\right)\right. \\
& \left.\oslash\left(\left[Q_{a u x}^{(a)} \odot A_{a u x}^{(a)}\right] V_{a u x}^{(a)} S_{a u x}^{(a)}+s \beta U_{a u x}^{(a)}\right)\right\},
\end{aligned}
$$

$V_{a u x}^{(a)} \leftarrow V_{a u x}^{(a)} \odot\left\{\left(R_{a u x}^{(a)}{ }^{\top} U_{a u x}^{(a)} S_{a u x}^{(a)}\right)\right.$

$$
\left.\oslash\left(\left[Q_{a u x}^{(a)} \odot A_{a u x}^{(a)}\right]^{\top} U_{a u x}^{(a)} S_{a u x}^{(a)}+s \beta V_{a u x}^{(a)}\right)\right\},
$$

$U_{\text {tgt }}^{(a)} \leftarrow U_{\text {tgt }}^{(a)} \odot\left\{\left(\left[R_{\text {tgt }}^{(t r a)}+\frac{\alpha}{s-1} \sum_{b=1, b \neq a}^{s} Q_{\text {tgt }}^{(v a l)} \odot A_{\text {tgt }}^{(b)}\right]\right.\right.$

$$
\left.\left.V_{\text {tgt }}^{(a)} S_{a u x}^{(a)}{ }^{\top}\right) \oslash\left(A_{\text {tgt }}^{(a)} V_{\text {tgt }}^{(a)} S_{a u x}^{(a)}{ }^{\top}+s \beta U_{\text {tgt }}^{(a)}\right)\right\},
$$

$$
\begin{aligned}
V_{\text {tgt }}^{(a)} \leftarrow & V_{\text {tgt }}^{(a)} \odot\left\{\left(\left[R_{\text {tgt }}^{(t r a)}+\frac{\alpha}{s-1} \sum_{b=1, b \neq a}^{s} Q_{\text {tgt }}^{(v a l)} \odot A_{\text {tgt }}^{(b)}\right]^{\top} U_{\text {tgt }}^{(a)} S_{\text {aux }}^{(a)}\right)\right. \\
& \left.\oslash\left(A_{\text {tgt }}^{(a)} U_{\text {tgt }}^{(a)} S_{\text {aux }}^{(a)}+s \beta V_{\text {tgt }}^{(a)}\right)\right\},
\end{aligned}
$$

$$
\begin{aligned}
& S_{\text {aux }}^{(a)} \leftarrow S_{\text {aux }}^{(a)} \odot\left\{\left(U_{\text {tgt }}^{(a)}\left[R_{\text {tgt }}^{(t r a)}+\frac{\alpha}{s-1} \sum_{b=1, b \neq a}^{s} Q_{\text {tgt }}^{(v a l)} \odot A_{\text {tgt }}^{(b)}\right] V_{\text {tgt }}^{(a)}\right.\right. \\
& \left.+U_{a u x}^{(a)^{\top}} R_{a u x}^{(a)} V_{a u x}^{(a)}\right) \\
& \left.\oslash\left(U_{a u x}^{(a)}{ }^{\top}\left[Q_{a u x}^{(a)} \odot A_{a u x}^{(a)}\right] V_{a u x}^{(a)}+U_{\text {tgt }}^{(a)^{\top}} A_{\text {tgt }}^{(a)} V_{\text {tgt }}^{(a)}+s \beta S_{a u x}^{(a)}\right)\right\} .
\end{aligned}
$$

With Eqs. (8) to (12), we can iteratively update $U_{a u x}^{(a)}, V_{a u x}^{(a)}$ $U_{\text {tgt }}^{(a)}, V_{\text {tgt }}^{(a)}$ and $S_{a u x}^{(a)}$, and the learning algorithm is illustrated in Algorithm 1. After the algorithm converges, we calculate the final predictions as follows,

$\widehat{R}_{\text {tgt }}=\frac{1}{s} \sum_{a=1}^{s} U_{\text {tgt }}^{(a)} S_{a u x}^{(a)} V_{\text {tgt }}^{(a)^{\top}}$.

Algorithm 1 Transfer collaborative filtering from multiple source domains via consensus regularization (TRACER).

Input: The rating data from source domains $R_{a u x}^{(1)}, \cdots, R_{\text {aux }}^{(s)}$, and the rating data for training in the target domain $R_{\text {tgt }}=R_{\text {tgt }}^{(\text {tra })} \cup R_{\text {tgt }}^{(\text {val })}$.

Parameters: The number of latent user clusters and item clusters in the ath source domain $k_{a u x}^{(a)}$ and $l_{a u x}^{(a)}(a=1, \cdots, s)$, the trade-off coefficients $\alpha$ and $\beta$, the max number of iterations $T$.

Define: The indicator matrix $Q_{a u x}^{(1)}, \cdots, Q_{a u x}^{(s)}$ and $Q_{t g t}^{(t r a)} \cup Q_{t g t}^{(v a l)}$ for the source domains and the target domain.

Output: The latent user clusters and item clusters $U_{a u x}^{(a)}, V_{a u x}^{(a)}, U_{\text {tgt }}^{(a)}, V_{\text {tgt }}^{(a)}$, and the rating patterns $S_{a u x}^{(a)}(a=1, \cdots, s)$.

1. for $a \leftarrow 1, \ldots, s$

2. Randomly initialize $U_{\text {aux }}^{(a)}, V_{\text {aux }}^{(a)}, U_{\text {tgt }}^{(a)}, V_{\text {tgt }}^{(a)}$ and $S_{\text {aux }}^{(a)}$;

3. end for

4. for $a \leftarrow 1, \ldots, s$

5. Update $U_{a u x}^{(a)}, V_{a u x}^{(a)}, U_{t g t}^{(a)}, V_{t g t}^{(a)}$ and $S_{a u x}^{(a)}$ according to Eqs. (8) to (12);

6. end for

7. Repeat Steps 4, 5, 6 until the algorithm converges, or the iterating number exceeds $T$.

\section{Experimental evaluation}

In this section, we first prepare two data sets from real-world applications, then we compare our algorithms with different baselines to validate the effectiveness of TRACER. The source code and experimental data sets of this work are available on https://github. com/hezi73/TRACER.

\subsection{Data preparation}

We crawl the data sets from the E-commerce websites Douban and Dianping, both of which are well known Chinese websites. 
Table 2

Details of Douban \& Dianping data sets.

\begin{tabular}{llll}
\hline Data set & Domain & \# of users/items/ratings & Density \\
\hline Douban & Movie & $10000 / 10000 / 5157078$ & $5.16 \%$ \\
& Music & $10000 / 10000 / 1394820$ & $1.39 \%$ \\
\multirow{3}{*}{ Dianping } & Book & $10000 / 10000 / 758087$ & $0.76 \%$ \\
& Shanghai & $5000 / 5000 / 952225$ & $3.81 \%$ \\
& Shenzhen & $5000 / 5000 / 264721$ & $1.06 \%$ \\
& Hangzhou & $5000 / 5000 / 605607$ & $2.42 \%$ \\
& Suzhou & $5000 / 5000 / 492753$ & $1.97 \%$ \\
& Beijing & $5000 / 5000 / 577665$ & $2.31 \%$ \\
\hline
\end{tabular}

- Douban data set. In this website, three domains of data including movie, music and book, are collected and preprocessed. For each domain, 10,000 users and 10,000 items are extracted, and the range of ratings is from 1 to 5 . Further, we define the data density as the ratio of ratings over (\#users $\times \#$ items) in a domain. The densities of these three data sets are respectively $5.16 \%, 1.39 \%$ and $0.76 \%$. To construct the TCF problems with multiple sources, we randomly select one domain as the target domain and the rest two are used as sources, therefore in this way we construct three TCF problems.

- Dianping data set. In this website, we focus on the task of restaurant recommendations, and we collect the data sets from five cities in China, including Shanghai, Shenzhen, Hangzhou, Suzhou and Beijing. For each domain the rating data of 5000 users rating on 5000 items are extracted, and the range of ratings is also from 1 to 5. The densities of these five domains are 3.81\%, 1.06\%, 2.42\%, 1.97\% and $2.31 \%$ respectively. Similar to Douban data set, we construct five TCF problems by randomly selecting one city as the target domain. Note that, here we just extract the same numbers of users and items to construct experimental data sets, while our framework does not need this condition.

In total, we construct 8 problems from these two data sets in the experiments. For clarity, the characteristics of these two data sets are summarized in Table 2.

\subsection{Experimental setting}

\subsubsection{Baselines}

To validate the effectiveness of our model TRACER, variable kinds of baselines are considered, including traditional collaborative filtering algorithms and transfer collaborative filtering ones.

- NMF (Non-negative matrix factorization) (Lee \& Seung, 2001): this is a non-negative matrix factorization algorithm for recommendation, which factorized the rating matrix into non-negative user and item latent factors.

- PMF (Probabilistic matrix factorization) (Mnih \& Salakhutdinov, 2008): this is a probabilistic matrix factorization for recommendation, which can obtain considerably good result for users with very few ratings.

- CBT (Codebook transfer) (Li et al., 2009): this is a transfer learning algorithm from a single source domain, which assumed that different domains share the rating pattern called codebook for knowledge transfer.

- CLFM (Cluster-level latent factor model) ${ }^{3}$ (Gao et al., 2013): this is also a TCF algorithm based on the cluster-level latent factor model, in which the codebook is further divided into two parts, i.e., common rating pattern shared across domains and domain-specific rating pattern, to improve the recommendation performance.

\footnotetext{
3 We would like to thank the authors of Gao et al. (2013) for providing their source code.
}

- TALMUD (Transfer learning for multiple domains) (Moreno et al., 2012): this method can handle multiple sources, which actually contained two steps. Specifically, they first conducted the CBT model, and then assigned different weights to the outputs from CBT, while our model combines the CBT model and consensus regularization into a unified framework. Most importantly, for the scores to be predicted, TALMUD assigns the same weight to each domain, while in our model, each source domain contributes differently for different predicted scores.

Implementation details. In the experiments, for each problem we randomly divide the rating data in the target domain into two parts, one for training and the rest for test. Specifically, we respectively sample $80 \%, 60 \%, 40 \%$ and $20 \%$ for training and the rest for test. In Section 4.3, we record the average results on three individual trials. On TRACER, we further divide the training data into two portions using ten-fold cross-validation, i.e., if we sample $80 \%$ for training, then $72 \%$ training data are used for learning from one single domain to one target domain, and $8 \%$ are treated as validation set. Therefore, we can get 10 outputs from ten-fold cross-validation. Finally, we integrate them together as the final result by assigning different weights which are derived based on the results of validation sets.

For TCF algorithms which only transfer knowledge from one source domain to one target domain, i.e., CBT and CLFM, we have $s$ outputs if there are $s$ source domains. Only the best results are reported for these methods. To simplify our model, we set $k_{a u x}^{(a)}=$ $k$ and $l_{a u x}^{(a)}=l(a=1, \ldots, s)$ for all source domains. Optimal parameters are set for all baselines. Specifically, for Dianping data set $k$ and $l$ are respectively set as $(100,100),(100,100),(100,100)$ in CBT, CLFM and TRACER. In CLFM, the sizes of common rating pattern and domain-specific rating pattern are the same. And the numbers of latent factors on users and items are set as 50, 10 for NMF and PMF.

\subsubsection{Evaluation metrics}

We use Mean Absolute Error (MAE) and Root Mean Square Error (RMSE), which are the most commonly used accuracy evaluation metrics in recommender systems, to evaluate the prediction performance of all algorithms.

$$
\begin{aligned}
\text { MAE } & =\frac{\sum_{(u, i) \in R_{\text {tgt }}^{(t e s t)}}\left|\left(R_{\text {tgt }}-\hat{R}_{\text {tgt }}\right)_{(u, i)}\right|}{\left|Q_{\text {tgt }}^{(\text {test })}\right|}, \\
\text { RMSE } & =\sqrt{\frac{\sum_{(u, i) \in R_{\text {tgt }}^{(t e s t)}}\left[\left(R_{\text {tgt }}-\hat{R}_{\text {tgt }}\right)_{(u, i)}\right]^{2}}{\left|Q_{\text {tgt }}^{(\text {test })}\right|}},
\end{aligned}
$$

where $R_{\text {tgt }}^{(\text {test })}$ denotes the test data in the target domain and $Q_{\text {tgt }}^{\text {(test) }}$ is the corresponding indicator matrix. Smaller values of MAE and RMSE mean better performance.

\subsection{Experimental results}

All the detailed results of 8 problems on two data sets are shown in Table 3 and Fig. 2. (Douban- $x$ and Dianping- $x$ mean that $x$ is used as the target domain.) From these results, we have following insightful observations,

- In most cases, TRACER outperforms all baselines, including both traditional collaborative filtering algorithms (NMF and PMF) and TCF algorithms (CBT, CLFM, TALMUD). It indicates the superiority of combining basic TCF framework with consensus regularization. 
Table 3

Detailed results on Douban-Book \& Dianping-Hangzhou (the improvement is based on NMF).

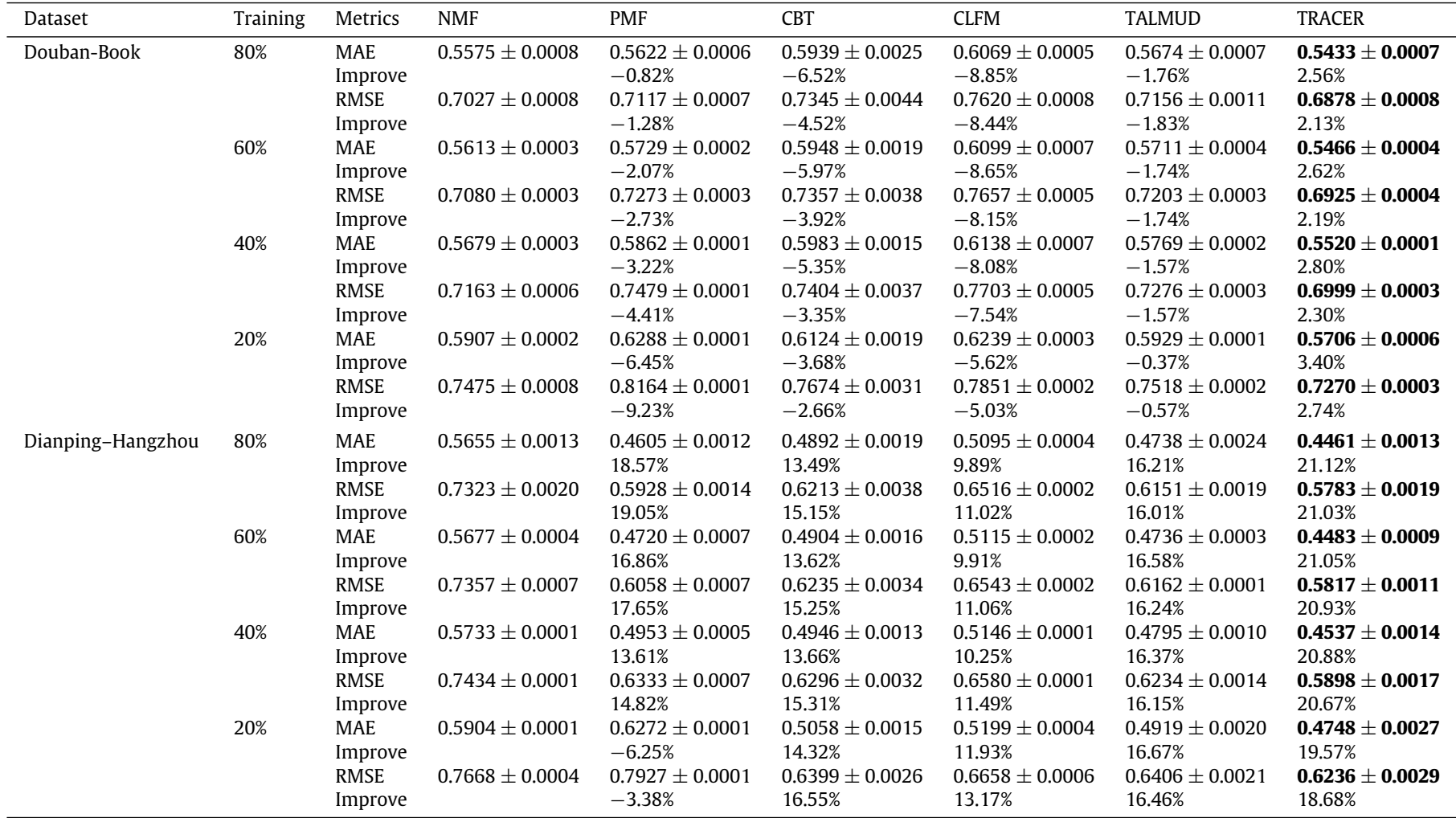
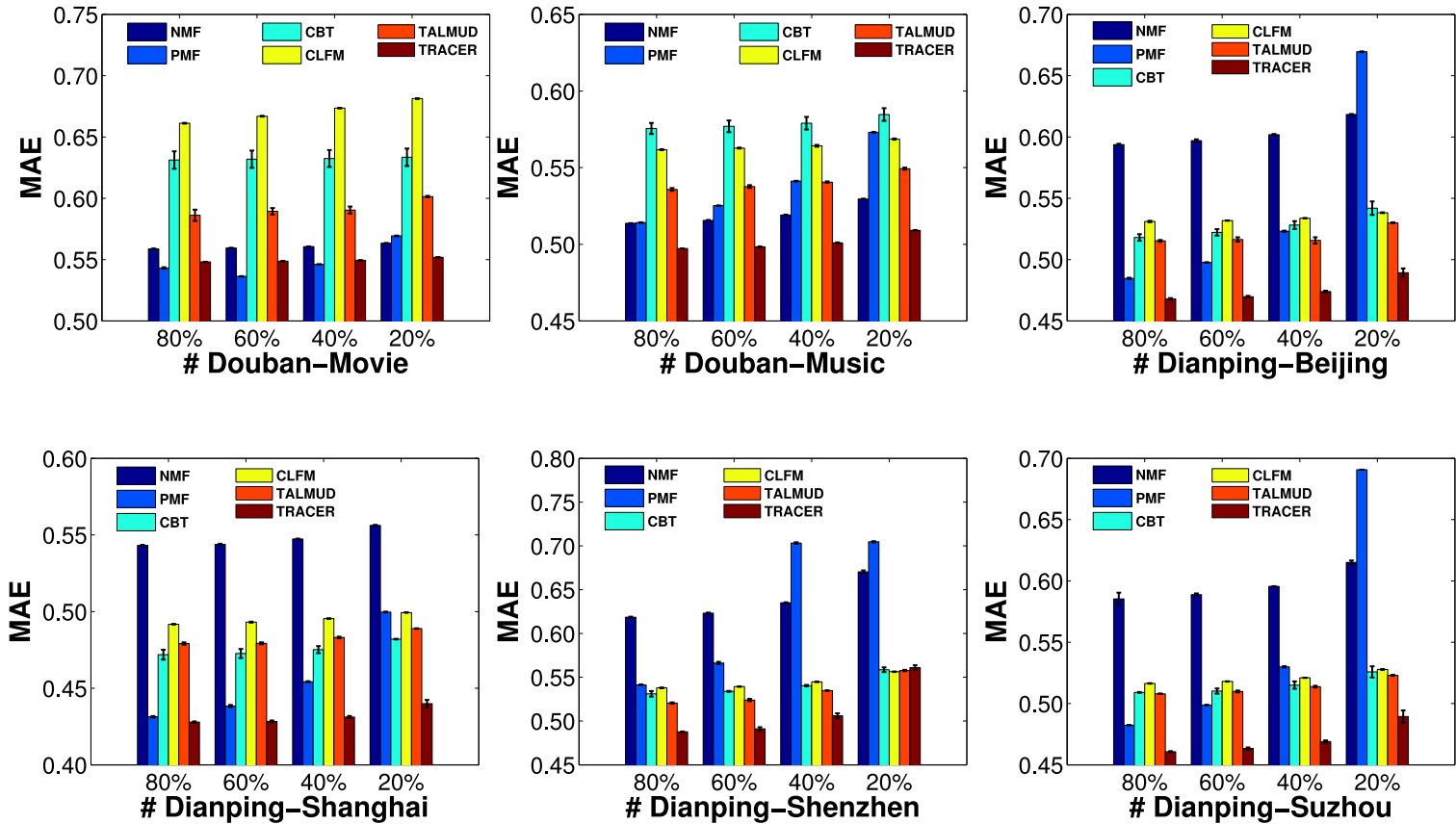

Fig. 2. The results on Douban \& Dianping.

- It seems that NMF and PMF can achieve relatively better performance than the baselines of TCF algorithms with abundant training data, especially on Douban data set. We analyze this phenomenon by visualizing the distributions of ratings on our data sets (i.e., Douban and Dianping), and find that the distributions of ratings are very unbalanced, especially on Douban. On Douban data set, the values of about $60 \%$ data are 5 , and the values of about $30 \%$ data are 4 . Therefore, we conjecture this reason leading to the poor performance of CBT, CLFM, TALMUD and the better performance of NMF and PMF. On Dianping data set, TCF methods can perform much more stable and better than NMF and PMF as the size of training data in the target domain become smaller. It shows that the TCF algorithms can learn knowledge from source domains to improve recommendation performance. 


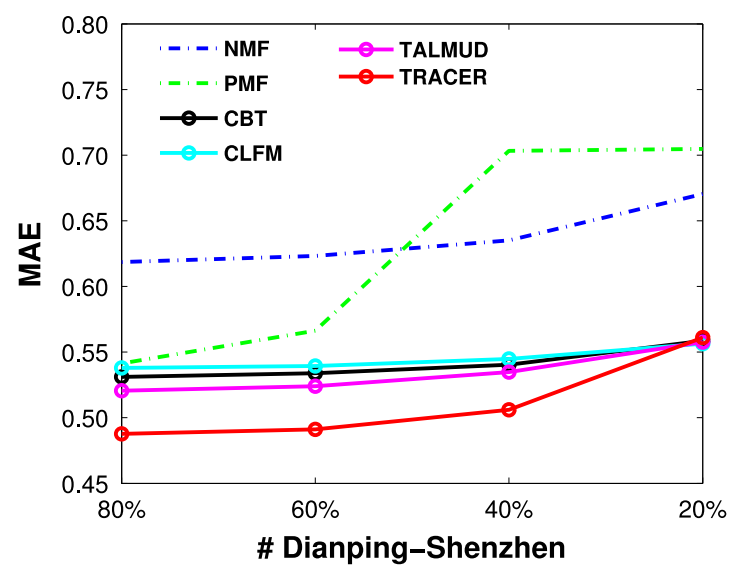

Fig. 3. Data-sparsity experimental results on Dianping data set.

- From these results, we also find that the previous TCF algorithms may not make full use of the knowledge from source domains, especially when training data are enough. Meanwhile, our model can effectively transfer the knowledge from the auxiliary domains, and obtain additional performance gain even with abundant training data, e.g., $80 \%$.

- TALMUD and TRACER perform better than other TCF algorithms (CBT and CLFM), which shows the necessity to learn from multiple source domains. We notice that TRACER does not outperform other TCF algorithms when 20\% data was used for training models on Dianping-Shenzhen. We analyze the experimental settings and find that the data in the Dianping-Shezhen is very sparse. Although the auxiliary data maybe enough, the training data on DianpingShenzhen is too sparse to reconstruct the rating matrix in the target domain when the training ratio is $20 \%$.

- Overall, TRACER can achieve the best results under different experimental settings of sampling for training (20\% 80\%), which shows the effectiveness of TRACER again.

\subsection{Exploration on sparsity data problem}

In the previous section, we validate the effectiveness of our model compared with the presentative CF and TCF methods. However, TRACER cannot work better than other TCF methods when training data is very sparse (e.g., $20 \%$ training data on DianpingShenzhen data set). Therefore, we explore the impact of data sparsity problem for rating prediction in this section. We perform the experiments on Dianping-Shenzhen problem with different training ratios $(80 \%, 60 \%, 40 \%, 20 \%)$.

The experimental results are shown in Fig. 3. The rating data of the target domain in this problem is quite sparse, which leads to poor performance of NMF and PMF, especially when training data become sparser. It is clear that TCF methods all achieve much stable and good performance, which demonstrates the superiority of TCF in alleviating data-sparsity problems and can benefit from transferring knowledge from auxiliary sources.

\subsection{Parameter investigation}

TRACER includes four parameters $(k, l, \alpha$ and $\beta$ ), which affect the performance of TRACER. $k$ and $l$ are the sizes of shared rating pattern, $\alpha$ controls the importance of consensus regularization and $\beta$ represents the regularization parameter to avoid over-fitting problem. We conduct this parameter investigation experiment on the Dianping-Beijing task.
When tuning two parameters, e.g., $k$ and $l$, the other two are fixed, e.g., $\alpha$ and $\beta$. The impacts of $k$ and $l$ on MAE and RMSE are shown in Fig. 4, and the impacts of $\alpha$ and $\beta$ are shown in Fig. 5. From Fig. 4, we find that when the values of $k$ and $l$ are both around 50 , TRACER obtains the best performance; when the values of $k$ and $l$ are large enough, the rating patterns tend to be rating matrices in source domains, which is unique in different domains and has no benefit for improvement; when the values of $k$ and $l$ are small, little useful information can be transferred for collaborative filtering. Therefore, $k$ and $l$ are both set as 50 in our experiments, which are not too large or too small.

In Fig. 5, we can see that the values of $\alpha$ have a significant influence on TRACER. $\alpha$ controls the importance of consensus regularization on multiple predicted outputs. When the value of $\alpha$ is large, the consensus regularization plays a leading role in the optimization process which would weaken the impact of transfer learning. When $\alpha$ is quite small, our method performs like the combination of 1 vs. 1 transfer learning method without constraints on outputs learned from multiple sources. For the parameter of $\beta$, our model can perform well when the values of $\beta$ are set small values, i.e., less than 0.1 . In order to get the optimal results on the Dianping-Beijing task, we set $\alpha$ as 1 and $\beta$ as 0.0001 .

\section{Conclusions}

In this paper, we take the advantage of transfer collaborative filtering methods in recommender systems and propose a transfer collaborative filtering framework from multiple sources via consensus regularization. Considering the different contributions of each source domain on the predicted ratings, we design the consensus regularization to force the prediction results generated from different source domains for the same missing rating to converge. In addition, in order to make the transfer learning process flexible and improve the recommendation performance, we model the learning process of TRACER in a uniform framework. Experiments on two real-world application data sets validate the effectiveness of TRACER.

\section{Acknowledgments}

This research work is supported by the National Key R\&D Program of China under Grant No. 2018YFB1004300, the National Natural Science Foundation of China under Grant Nos. 61773361, 61473273, 91546122, the Science and Technology Project of Guangdong Province under Grant No. 2015B010109005, the Project of Youth Innovation Promotion Association CAS under Grant No. 2017146.

\section{Appendix}

To simplify the math expressions, we introduce two intermediate variables.

$A_{\text {aux }}^{(a)}=U_{\text {aux }}^{(a)} S_{a u x}^{(a)} V_{\text {aux }}^{(a)} \top^{\top}, \quad A_{\text {tgt }}^{(a)}=U_{\text {tgt }}^{(a)} S_{a u x}^{(a)} V_{\text {tgt }}^{(a)} \top^{\top}$,

where $a=1, \ldots, s$. Firstly, we can calculate the partial derivatives of $J_{1}$ with respect to the variables of $U_{a u x}^{(a)}, V_{a u x}^{(a)}, U_{t g t}^{(a)}, V_{\text {tgt }}^{(a)}$ and $S_{a u x}^{(a)}$ as follows,

$$
\begin{aligned}
\frac{\partial J_{1}}{\partial U_{a u x}^{(a)}} & =\frac{2}{s}\left(\left[Q_{a u x}^{(a)} \odot A_{a u x}^{(a)}\right] V_{a u x}^{(a)} S_{a u x}^{(a)} \top^{\top}-R_{a u x}^{(a)} V_{a u x}^{(a)} S_{a u x}^{(a)}{ }^{\top}\right), \\
\frac{\partial J_{1}}{\partial V_{a u x}^{(a)}} & =\frac{2}{s}\left(\left[Q_{a u x}^{(a)} \odot A_{a u x}^{(a)}\right]^{\top} U_{a u x}^{(a)} S_{a u x}^{(a)}-R_{a u x}^{(a)}{ }^{\top} U_{a u x}^{(a)} S_{a u x}^{(a)}\right), \\
\frac{\partial J_{1}}{\partial U_{t g t}^{(a)}} & =\frac{2}{s}\left(\left[Q_{t g t}^{(t r a)} \odot A_{t g t}^{(a)}\right] V_{t g t}^{(a)} S_{a u x}^{(a)}{ }^{\top}-R_{\text {tgt }}^{(t r a)} V_{\text {tgt }}^{(a)}\left[S_{a u x}^{(a)}\right]^{\top}\right),
\end{aligned}
$$




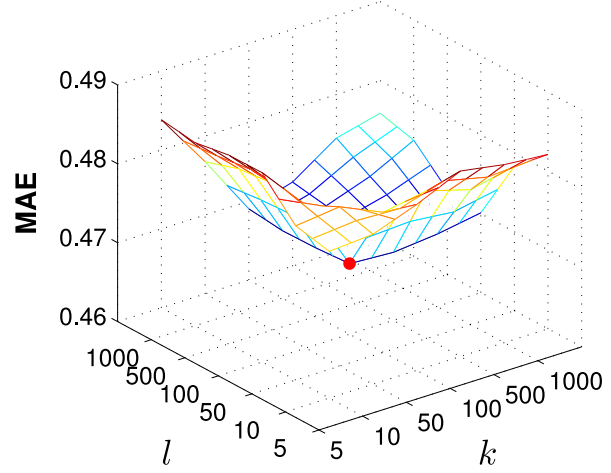

(a) MAE

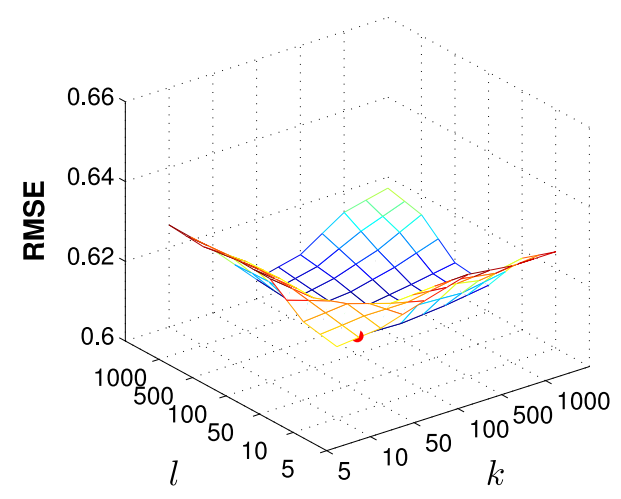

(b) RMSE

Fig. 4. Parameter study on $k$ and $l$.

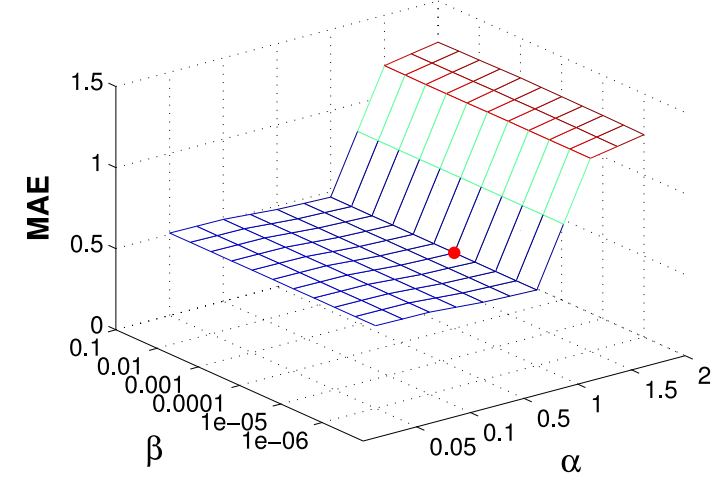

(a) MAE

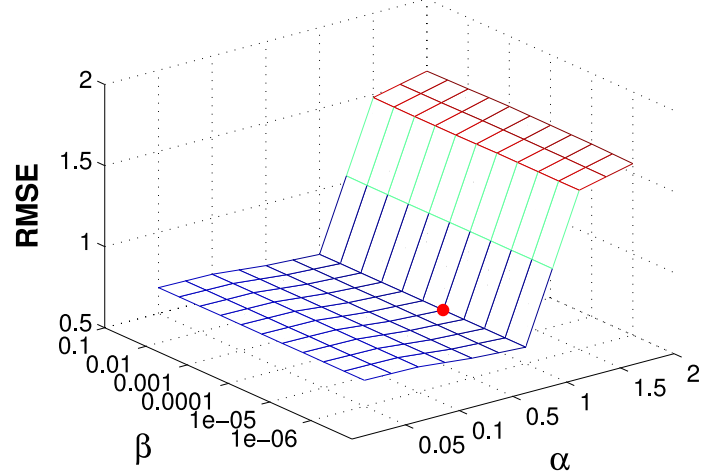

(b) RMSE

Fig. 5. Parameter study on $\alpha$ and $\beta$.

$$
\begin{aligned}
\frac{\partial J_{1}}{\partial V_{\text {tgt }}^{(a)}}= & \frac{2}{S}\left(\left[Q_{\text {tgt }}^{(t r a)} \odot A_{\text {tgt }}^{(a)}\right]^{\top} U_{\text {tgt }}^{(a)} S_{a u x}^{(a)}-R_{\text {tgt }}^{(t r a)^{\top}} U_{\text {tgt }}^{(a)} S_{a u x}^{(a)}\right), \\
\frac{\partial J_{1}}{\partial S_{a u x}^{(a)}}= & \frac{2}{S}\left(U_{a u x}^{(a)}{ }^{\top}\left[Q_{a u x}^{(a)} \odot A_{a u x}^{(a)}\right] V_{a u x}^{(a)}-U_{a u x}^{(a)}{ }^{\top} R_{a u x}^{(a)} V_{a u x}^{(a)}\right. \\
& \left.+U_{\text {tgt }}^{(a)^{\top}}\left[Q_{\text {tgt }}^{(t r a)} \odot A_{\text {tgt }}^{(a)}\right] V_{\text {tgt }}^{(a)}-U_{\text {tgt }}^{(a)^{\top}} R_{\text {tgt }}^{(a)} V_{\text {tgt }}^{(a)}\right) .
\end{aligned}
$$

The partial derivatives of $J_{2}$ with respect to the variables of $U_{\text {tgt }}^{(a)}, V_{t g t}^{(a)}$ and $S_{\text {aux }}^{(a)}$ are as follows,

$$
\begin{aligned}
& \frac{\partial J_{2}}{\partial U_{\text {tgt }}^{(a)}}=\frac{2}{S}\left[Q_{\text {tgt }}^{(v a l)} \odot A_{\text {tgt }}^{(a)}\right] V_{\text {tgt }}^{(a)} S_{a u x}^{(a)} \top^{\top} \\
& -\frac{2}{s(s-1)} \sum_{b=1, b \neq a}^{s}\left[Q_{\text {tgt }}^{(v a l)} \odot A_{\text {tgt }}^{(b)}\right] V_{\text {tgt }}^{(a)} S_{a u x}^{(a)^{\top}}, \\
& \frac{\partial J_{2}}{\partial V_{t g t}^{(a)}}=\frac{2}{s}\left[Q_{t g t}^{(v a l)} \odot A_{t g t}^{(a)}\right]^{\top} U_{t g t}^{(a)} S_{a u x}^{(a)} \\
& -\frac{2}{s(s-1)} \sum_{b=1, b \neq a}^{s}\left[Q_{t g t}^{(v a l)} \odot A_{t g t}^{(b)}\right]^{\top} U_{t g t}^{(a)} S_{a u x}^{(a)}, \\
& \frac{\partial J_{2}}{\partial S_{a u x}^{(a)}}=\frac{2}{S} U_{t g t}^{(a)^{\top}}\left[Q_{\text {tgt }}^{v a l} \odot A_{t g t}^{(a)}\right] V_{\text {tgt }}^{(a)} \\
& -\frac{2}{s(s-1)} U_{\text {tgt }}^{(a)^{\top}} \sum_{b=1, b \neq a}^{s}\left[Q_{\text {tgt }}^{(v a l)} \odot A_{\text {tgt }}^{b}\right] V_{\text {tgt }}^{(a)} .
\end{aligned}
$$

The partial derivatives of $J_{3}$ with respect to the variables of $U_{a u x}^{(a)}$, $V_{a u x}^{(a)}, U_{\text {tgt }}^{(a)}, V_{\text {tgt }}^{(a)}$ and $S_{\text {aux }}^{(a)}$ are as follows,

$$
\begin{aligned}
& \frac{\partial J_{3}}{\partial U_{a u x}^{(a)}}=2 U_{a u x}^{(a)}, \frac{\partial J_{3}}{\partial V_{a u x}^{(a)}}=2 V_{a u x}^{(a)}, \\
& \frac{\partial J_{3}}{\partial U_{\text {tgt }}^{(a)}}=2 U_{\text {tgt }}^{(a)}, \frac{\partial J_{3}}{\partial V_{\text {tgt }}^{(a)}}=2 V_{\text {tgt }}^{(a)}, \frac{\partial J_{3}}{\partial S_{a u x}^{(a)}}=2 S_{a u x}^{(a)} .
\end{aligned}
$$

To minimize the objective $J$, the partial derivatives can be computed as follows,

$\frac{\partial J}{\partial U_{\text {aux }}^{(a)}}=\frac{\partial J_{1}}{\partial U_{\text {aux }}^{(a)}}+\beta \frac{\partial J_{3}}{\partial U_{\text {aux }}^{(a)}}$,

$\frac{\partial J}{\partial V_{a u x}^{(a)}}=\frac{\partial J_{1}}{\partial V_{a u x}^{(a)}}+\beta \frac{\partial J_{3}}{\partial V_{a u x}^{(a)}}$,

$\frac{\partial J}{\partial U_{t g t}^{(a)}}=\frac{\partial J_{1}}{\partial U_{\text {tgt }}^{(a)}}+\alpha \frac{\partial J_{2}}{\partial U_{\text {tgt }}^{(a)}}+\beta \frac{\partial J_{3}}{\partial U_{t g t}^{(a)}}$,

$\frac{\partial J}{\partial V_{\text {tgt }}^{(a)}}=\frac{\partial J_{1}}{\partial V_{\text {tgt }}^{(a)}}+\alpha \frac{\partial J_{2}}{\partial V_{\text {tgt }}^{(a)}}+\beta \frac{\partial J_{3}}{\partial V_{\text {tgt }}^{(a)}}$,

$\frac{\partial J}{\partial S_{a u x}^{(a)}}=\frac{\partial J_{1}}{\partial S_{a u x}^{(a)}}+\alpha \frac{\partial J_{2}}{\partial S_{a u x}^{(a)}}+\beta \frac{\partial J_{3}}{\partial S_{a u x}^{(a)}}$.

Based on the partial derivatives, we can develop following updating rules for $U_{a u x}^{(a)}, V_{a u x}^{(a)}, U_{\text {tgt }}^{(a)}, V_{\text {tgt }}^{(a)}$ and $S_{a u x}^{(a)}$ with Eqs. (8) to (12). 


\section{References}

Bobadilla, J., Ortega, F., \& Hernando, A. (2013). Recommender systems survey. Knowledge-Based Systems, 46(1), 109-132.

Chang, W.-C., Wu, Y., Liu, H., \& Yang, Y. (2017). Cross-Domain kernel induction for transfer learning. In Proceedings of the 31st AAAI conference on artificial intelligence (pp. 1763-1769).

Diao, Q., Qiu, M., Wu, C.-Y., Smola, A. J., Jiang, J., \& Wang, C. (2014). Jointly modeling aspects, ratings and sentiments for movie recommendation (jmars). In Proceedings of the 20th ACM SIGKDD international conference on knowledge discovery and data mining (pp. 193-202). ACM.

Fernández-Tobías, I., Cantador, I., Kaminskas, M., \& Ricci, F. (2012). Cross-domain recommender systems: A survey of the state of the art. In Spanish conference on information retrieval (pp. 24-35). SN.

Fu, Y., Liu, B., Ge, Y., Yao, Z., \& Xiong, H. (2014). User preference learning with multiple information fusion for restaurant recommendation. In Proceedings of the 2014 SIAM international conference on data mining (pp. 470-478). SIAM.

Gao, S., Luo, H., Chen, D., Li, S., Gallinari, P., \& Guo, J. (2013). Cross-domain recommendation via cluster-level latent factor model. In Joint European conference on machine learning and knowledge discovery in databases (pp. 161-176). Springer.

Gu, Y., Sun, Y., \& Gao, J. (2017). The co-evolution model for social network evolving and opinion migration. In Proceedings of the 23rd ACM SIGKDD international conference on knowledge discovery and data mining (pp. 175-184). ACM.

He, X., Chen, T., Kan, M.-Y., \& Chen, X. (2015). Trirank: review-aware explainable recommendation by modeling aspects. In Proceedings of the 24th ACM international conference on information and knowledge management (pp. 1661-1670). ACM.

He, R., \& McAuley, J. (2016). Ups and downs: modeling the visual evolution of fashion trends with one-class collaborative filtering. In Proceedings of the 25th international conference on world wide web (pp. 507-517).

Hernando, A., Bobadilla, J., \& Ortega, F. (2016). A non negative matrix factorization for collaborative filtering recommender systems based on a bayesian probabilistic model. Knowledge-Based Systems, 97, 188-202.

Hong, L., Doumith, A. S., \& Davison, B. D. (2013). Co-factorization machines: modeling user interests and predicting individual decisions in twitter. In Proceedings of the 6th ACM international conference on web search and data mining (pp. 557-566). ACM.

Jamali, M., \& Lakshmanan, L. (2013). Heteromf: recommendation in heterogeneous information networks using context dependent factor models. In Proceedings of the 22nd international conference on world wide web (pp. 643-654). ACM.

Jiang, M., Cui, P., Yuan, N. J., Xie, X., \& Yang, S. (2016). Little is much: bridging crossplatform behaviors through overlapped crowds. In Proceedings of the 30th AAAI conference on artificial intelligence (pp. 13-19).

Kawamae, N. (2015). Real time recommendations from connoisseurs. In Proceedings of the 21 th ACM SIGKDD international conference on knowledge discovery and data mining (pp. 537-546). ACM.

Lee, D. D., \& Seung, H. S. (2001). Algorithms for non-negative matrix factorization. In Advances in neural information processing systems (pp. 556-562).
Li, J., Guo, B., Wang, Z., Li, M., \& Yu, Z. (2016). Where to place the next outlet? harnessing cross-space urban data for multi-scale chain store recommendation. In Proceedings of the 2016 ACM international joint conference on pervasive and ubiquitous computing: adjunct (pp. 149-152). ACM.

Li, B., Yang, Q., \& Xue, X. (2009). Can movies and books collaborate? cross-domain collaborative filtering for sparsity reduction. In Proceedings of the 21st international joint conference on artificial intelligence (pp. 2052-2057).

Lian, D., Zhao, C., Xie, X., Sun, G., Chen, E., \& Rui, Y. (2014). Geomf: joint geographical modeling and matrix factorization for point-of-interest recommendation. In Proceedings of the 20th ACM SIGKDD international conference on knowledge discovery and data mining (pp. 831-840). ACM.

Ma, H., King, I., \& Lyu, M. R. (2009). Learning to recommend with social trust ensemble. In International ACM SIGIR conference on research and development in information retrieval (pp. 203-210).

Mnih, A., \& Salakhutdinov, R. R. (2008). Probabilistic matrix factorization, In Advances in neural information processing systems (pp. 1257-1264).

Moreno, O., Shapira, B., Rokach, L., \& Shani, G. (2012). Talmud: transfer learning for multiple domains. In Proceedings of the 21st ACM international conference on information and knowledge management (pp. 425-434). ACM.

Pan, S. J., \& Yang, Q. (2010). A survey on transfer learning. IEEE Transactions on Knowledge and Data Engineering, 22(10), 1345-1359.

Ricci, F., Rokach, L., \& Shapira, B. (2011). Introduction to recommender systems handbook. In Recommender systems handbook (pp. 1-35). Springer.

Shi, Y., Larson, M., \& Hanjalic, A. (2013). Generalized tag-induced cross-domain collaborative filtering CoRR, abs/1302.4888.

Shi, Y., Larson, M., \& Hanjalic, A. (2014). Collaborative filtering beyond the user-item matrix: a survey of the state of the art and future challenges. ACM Computing Surveys, 47(1), 3:1-3:45.

Shi, C., Zhang, Z., Luo, P., Yu, P. S., Yue, Y., \& Wu, B. (2015). Semantic path based personalized recommendation on weighted heterogeneous information networks. In Proceedings of the 24th ACM international conference on information and knowledge management (pp. 453-462). ACM.

Srebro, N., \& Jaakkola, T. (2003). Weighted low-rank approximations. In Proceedings of the 20th international conference on machine learning (pp. 720-727).

Wang, Y., Yuan, N. J., Lian, D., Xu, L., Xie, X., Chen, E., et al. (2015). Regularity and conformity: Location prediction using heterogeneous mobility data. In Proceedings of the 21 th ACM SIGKDD international conference on knowledge discovery and data mining (pp. 1275-1284). ACM.

Yang, B., Lei, Y., Liu, J., \& Li, W. (2017). Social collaborative filtering by trust. IEEE Transactions on Pattern Analysis and Machine Intelligence, 39(8), 1633-1647.

Ye, M., Yin, P., Lee, W.-C., \& Lee, D.-L. (2011). Exploiting geographical influence for collaborative point-of-interest recommendation. In Proceedings of the 34th international ACM SIGIR conference on research and development in information retrieval (pp. 325-334). ACM.

Yu, F., Che, N., Li, Z., Li, K., \& Jiang, S. (2017). Friend recommendation considering preference coverage in location-based social networks. In Pacific-Asia conference on knowledge discovery and data mining (pp. 91-105). Springer.

Zhuang, F., Luo, P., Xiong, H., Xiong, Y., He, Q., \& Shi, Z. (2010). Cross-domain learning from multiple sources: A consensus regularization perspective. IEEE Transactions on Knowledge and Data Engineering, 22(12), 1664-1678. 